\title{
Effects of graphene polymer nano composite coating on corrosion resistance of ASTM A106 carbon steel pipe
}

\author{
Adiba Rhaodah Andsaler a, Wilver Philip a , Izman Sudin a , Mohd Zamri Mohd Yusop a,b, \\ a School of Mechanical Engineering, Faculty of Engineering, Universiti Teknologi Malaysia, 81310 UTM Skudai, Johor, Malaysia \\ b Advanced Membrane Technology and Research Centre, Universiti Teknologi Malaysia, 81310 Johor Bahru, Malaysia \\ * Corresponding author: zamriyusop@utm.my
}

\section{Article history}

Received 30 September 2019

Revised 22 December 2019

Accepted 21 January 2020

Published Online 26 August 2020

\begin{abstract}
Wide application prospects of graphene in the corrosion protection coatings field are owing to its excellent mechanical properties, outstanding chemical stability, corrosion resistance, and high corrosion resistance. Anti-corrosion composite was prepared using multilayer graphene powder as a filler and epoxy resin in this work to investigate the effect of graphene compositions in the zinc-rich epoxy (ZRE) as the modified anti-corrosion coating for carbon steel pipe. Using SEM for morphology along with the electrochemical performance was associated with changed content composite coating of graphene and pure epoxy resin coating. The measurement for open-circuit potential (OCP), the potentiostat polarization curve (Tafel Plot), and the electrochemical impedance spectroscopy (EIS) of the coating were conducted in order to investigate the influence of additional of graphene on the basic properties and corrosion resistance. From the experimental results, the increasing content of graphene led by the increasing value of OCP as the test system was stable, also the coating of high content graphene sustained a moderately high OCP ability with the increase of immersion time. From the results in the industrial environment, $0.1 \mathrm{wt} \%$ Graphene $+99.9 \mathrm{wt} \%$ ZRE in a $240 \mu \mathrm{m}$ coating layer would be sufficient as the corrosion rate is $0.0087204 \mathrm{mmpy}$ and three times lower compare to $100 \mathrm{wt} \%$ ZRE. In a nutshell, the addition of graphene improves the impenetrability of the composite coating.
\end{abstract}

Keywords: Graphene composite, anti-corrosion coating, zinc-rich epoxy, open circuit potential, electrochemical impedance spectroscopy

(C) 2020 Penerbit UTM Press. All rights reserved

\section{INTRODUCTION}

Thermosetting polymer, epoxy resin [1-3] has outstanding performances as a crucial material source in global industries in which broadly used in aerospace, automotive, construction, and marine chemical industry [4-6]. Nowadays, most industrial materials heavily rely on steel products resulting in the critical corrosion problem in a certain environment. As a result, better corrosion protection materials are demanded interminably due to more steel products were applied at an extreme environment such as offshore and ports which leaving the current conventional epoxy coating protection worthless to shield these steels in those extreme environmental requirements [7]. Currently, a few studies on corrosion protection have been demonstrated through the variation materials of epoxy resin and additional high-performance anti-corrosion filler which show more enhancement on anti-corrosion properties [8-10]. However, there are not many reports on using carbon materials such as graphene, carbon nanotubes, carbon fibers, and fullerene as the nanofiller in metal embedded corrosion protection coating that giving detailed results on EIS and Tafel.

Graphene is a two-dimensional nanomaterial-based from carbon element is the toughest acknowledged material $[11,12]$. This material comprises of a planar monolayer of carbon atoms with the $\mathrm{C}=\mathrm{C}$ bond length of $0.142 \mathrm{~nm}[13,14]$. It was first extracted at Manchester University in 2004 as a bounded by sp2 of carbon atom with singleatom layer crystal $[6,15]$. In terms of application, it is used in the corrosion protection coatings technology for its outstanding chemical stability, high conductivity, and excellent mechanical properties $[5,6,16]$. In order to prevent diffusion and permeation, constant graphene layer structure tolerates constant physical barrier between base metal and the active medium. A test on graphene sealed a small "balloon" found that graphene has a good thermal and chemical stability for under extremely high temperature $\left(>1500^{\circ} \mathrm{C}\right)$ condition or in the corrosive gas and liquid environment can efficiently hinder the channel of any gas atoms. Graphene still can sustain stability proving it is an excellent protective coating material [17,18].

Most of malfunctions for incidents application as such oil and gas pipelines are resulted from corrosion and corrosion-induced damage [17]. The main reason is exposure of corrosion attack as oil and gas pipelines are generally made from low-carbon steel $[19,20]$. To prevent direct contact between metals and corrosive agents, protective film using polymeric coating is broadly used [19]. The exposure of aggressive environments by steel pipelines as such abrasion, hydrothermal aging, and chemical degradation. These corrosive environments generally degrade the protective coatings and reduce their ability on corrosion protection gradually [21]. Due to cheaper price, good resistance of corrosion, and bond to substrates, corrosion protection film using epoxy coating for metallic structures is preferred $[22,23]$. Liu et al. made-up the coating of graphene/epoxy with two different weight composition of graphene, 0.5 and $1.0 \mathrm{wt} \%$ resulting the coating with $0.5 \mathrm{wt} . \%$ graphene produced well performance and the presence of graphene nanoparticles which enhanced the epoxy coating the corrosion resistance [15]. Monetta et al. assessed the coating of graphene/epoxy with $1.0 \mathrm{wt} . \%$ of graphene nanoparticles 
performances in which indicated the graphene greatly enhanced the polymers anticorrosion properties [14]. Also, the addition of graphene does not affect the epoxy curing process and no significant changes found in water repellency and adhesion of cured coating.

In a different work, $\mathrm{Yu}$ et al. stated that a graphene with high content of nanoparticles still provide high resistance to gas and corrosion barrier. They concluded that graphene with $2.0 \mathrm{wt} . \%$ of nanoparticles additions reached a great enhancement on performance of anti-corrosion and gas barrier properties by evaluation of graphene from 0.5 to $2.0 \mathrm{wt} . \%$ [16]. Here we know that graphene displays its great capability for systems of corrosion coating. For each study has different focuses, some of the conclusion may differ with each other. Also, it is still in contentious in obtaining strong reinforcement for coating of nanocomposite with how much of graphene needed. [17].

In this paper, a multilayer of graphene and epoxy resin was used for the experiment. Graphene/epoxy with different proportions were prepared by changing the graphene amount. ASTM A106 Gr B carbon steel grade was chosen as the steel material due to the vast application in the offshore piping system for the crude oils. The consistency and homogeneity of graphene in coating system were enhanced using an ultrasonic sonicator by mixing the solution. Open circuit potential (OCP), the potentiostat polarization curve (Tafel Plot), and the electrochemical impedance spectroscopy (EIS) of the coating were analyzed in detail. The effect for nanofillers of graphene on the coating properties, resistance corrosion, and the coating mechanism of corrosion will be explained in the discussion section.

\section{EXPERIMENTAL}

\section{Materials}

The steel pipe samples substrate was supplied by Petronas Carigali Sdn Bhd - Sarawak Oil for research purposes. The properties of the sample used are carbon steel with material grade ASTM A106 GR.B and the predominant pipe type is a seamless pipe. The samples then undergone fabrication work to obtain the desired dimension for the corrosion test. A wire-cut Electrical Discharge Machine (EDM) was used to cut the samples into a square shape and CNC Milling Machine was used to mill the samples from $6 \mathrm{~mm}$ into $4 \mathrm{~mm}$ thickness. The dimension of the finished sample is $20 \mathrm{~mm}$ (length) $\mathrm{x}$ $20 \mathrm{~mm}$ (width) $\times 4 \mathrm{~mm}$ (thickness). To polish the surface of the samples, sandblasting and silicon carbide grinding paper were used as post treatments.

\section{Coating preparation}

The paint used in this experiment was a Zinc-Rich Polymer paint which was chosen according to the PETRONAS Technical Standard Protective Coatings and Linings. The addition of graphene powder into the paint solution was done by the weight-age ratio. The composition of the paint solution is $100 \mathrm{wt} \%$ of the zinc-rich polymer, $99.9 \mathrm{wt} \%$ of zinc-rich polymer with $0.1 \mathrm{wt} \%$ of graphene, $99.7 \mathrm{wt} \%$ of zinc-rich polymer with $0.3 \mathrm{wt} \%$ of graphene, and $99.5 \mathrm{wt} \%$ of zinc-rich polymer with $0.5 \mathrm{wt} \%$ of graphene. In order to get a homogeneous solution after adding graphene powder into the paint solution, the solution was sonicated for 10 minutes before the coating stage.

The coating layers in this experiment were determined by referring to the standard used in PETRONAS Technical Standard - Protective Coatings and Linings given by Petronas Carigali Sdn. Bhd. According to the standard, the primer layer consists of zinc-rich primers that shall be overcoated with chemically resistant coatings to avoid direct exposure acid and strong alkalis such as in the extreme environment. In this experiment, the standard stated that the primer layer was fixed with $240 \mu \mathrm{m}$.

A thin film coater was used in order to do the coating. To obtain a $240 \mu \mathrm{m}$ coating layer, a $10 \mu \mathrm{m}$ shim plate was added to the base of the coating plate and a $250 \mu \mathrm{m}$ thin-film coater was used. After the coating process was done, the samples were left to dry for 21 days at room temperature before proceed to composition analysis and corrosion test.

\section{Electrochemical performance test of coating}

The operating software used was Power Suite, as Princeton Applied Research Company made the PARSTAT 2273 electrochemical workstation. There are three-electrode system of electrochemical adopted, which the sample coating as working electrode, opposite electrode as platinum film electrode and saturated calomel electrode as the reference electrode. The corrosion liquid electrolyte used $3.5 \mathrm{wt} \% \mathrm{NaCl}$ solution, the environment temperature test was kept at $24^{\circ} \mathrm{C}$ (room temperature) and before the electrochemical testing, the workstation needs to be preheated for 10 minutes.

\section{Open circuit potential (OCP) test}

Before the OCP test, the electrolyte solution was immersed by the working electrode for 24 hours in order to ensure the test stability. The observation of OCP changes in the last 300 s as the time of scan is set temporary at $3600 \mathrm{~s}$, and the speed is set as $1 \mathrm{~s} /$ point. The OCP value for time period was taken in average if the value was less than $5 \mathrm{mV}$, then Origin software is used to calculate the trend of OCP with the time of immersion.

\section{Tafel plot test}

With the sample working area of $1 \mathrm{~cm}^{2}$ and $1.0 \mathrm{mV} / \mathrm{s}$ set for speed of scan, the scanning range of electro-kinetic potential is set from $0.3 \mathrm{~V}$ (vs OCP) to $1.5 \mathrm{~V}$ (vs OCP). Power Suite software was used to calculate the potential corrosion and the current density of corrosion after the calculation of complete polarization curve.

\section{Electrochemical Impedance Spectroscopy (EIS) test}

The penetration of sample in the $3.5 \mathrm{wt} \% \mathrm{NaCl}$ solution then the sample determination of electrochemical impedance spectra. To obtain the fitting parameters of impedance spectrum, ZSimpWin software was used for analysis of measuring the Nyquist and Bode map of impedance spectrum, while the scanning range frequency set at $100 \mathrm{mHz}-100 \mathrm{kHz}$ and the applied sine wave signal amplitude set at $10 \mathrm{mV}$.

\section{RESULTS AND DISCUSSION}

\section{Composition analysis}

The coating layer and composition used for the test were $100 \mathrm{wt} \%$ pristine zinc-rich polymer, $99.9 \mathrm{wt} \%$ of zinc-rich polymer with $0.1 \mathrm{wt} \%$ of graphene, $99.7 \mathrm{wt} \%$ of zinc-rich polymer with $0.3 \mathrm{wt} \%$ of graphene, and $99.5 \mathrm{wt} \%$ of zinc-rich polymer with $0.5 \mathrm{wt} \%$ of graphene along with $240 \mu \mathrm{m}$ coating layer being fixed. Fig. 1 shows scanning electron micrograph of the top view of the samples in which indicates the small presence of graphene in Fig.1(b), (c), (d) and (e) along with the totally zinc-rich polymer in Fig.1(a) in which showed in round mass structure. The morphological surface is shown by using Field Emission Scanning Electron Microscope (FESEM). Meanwhile, inside the Scanning Electron Microscope (SEM) vacuum chamber, the electron bombarded into the surface of the samples, then as the heat transfer to the particles, the zinc-rich polymer will agglomerate in which make them form in a round mass structure in Fig.1(b)-(e).

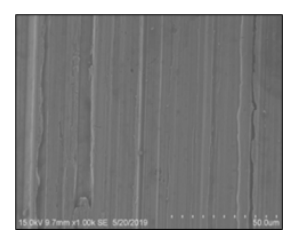

(a)

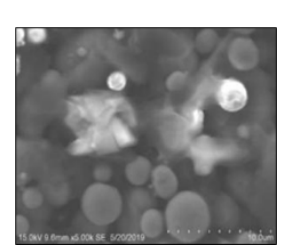

(d)

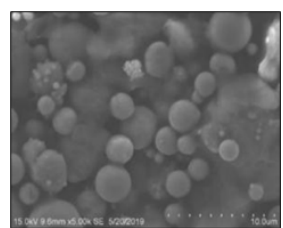

(b)

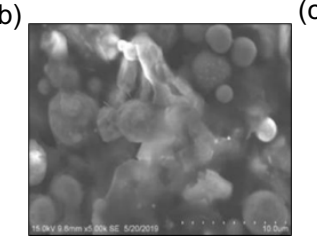

(e)
Fig. 1 Scanning electron micrograph of top view of uncoated sample (a), coated sample of $100 w t \%$ Pristine Zinc-Rich Polymer (Round Mass Structure) (b), 0.1wt\% Graphene (c), 0.3wt\% Graphene (d), and 0.5wt\% Graphene (e), (Graphene present as Flake Likes Structure) 


\section{Analysis of Open circuit potential (OCP)}

For the OCP analysis, a graph on potential vs elapsed time by using a $240 \mu \mathrm{m}$ coating layer is plotted in Fig. 2. The OCP test for coated sample in solution of $3.5 \mathrm{wt} \% \mathrm{NaCl}$ in a long time due to the value of OCP changed nonstop. When the stability of the test system was detected, thus the open circuit potential as shown from experimental results. Also, the test system stable lead to OCP value increase with the increasing content of graphene as well as the increase of immersion time, along with the coating of graphene with high content sustained a relatively high OCP ability.

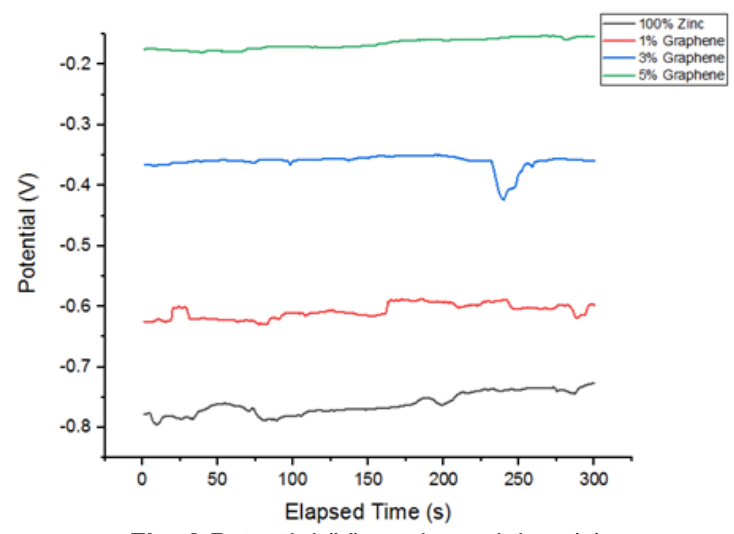

Fig. 2 Potential (V) vs elapsed time (s).

From the basic knowledge of metal corrosion, the electrochemical corrosion may occur when potential metal oxidation equilibrium is lower than potential reduction reaction of electrolyte balance. The stability of electrochemical properties can be maintained as the corrosion tendency of potential difference greater as well as open circuit potential higher. Hence, graphene coating can lessen the possibility of electrochemical corrosion.

\section{Analysis of Tafel plot}

Fig. 3 shows the comparison for polarization curves of coatings in different graphene content, which appearing in the anodic polarization curve as stable passivation region. This indicates the formation of compact oxidation protective film on the stainless-steel surface, then great inhibition of anodic dissolution reaction thus slowing down the corrosion rate. The comparison of electrochemical of coating parameters and the results for evaluation of coating corrosion resistance shown in Table 1. From the results, the corrosion current of steel substrates (Icorr) can be reduce effectively by coating, while the corrosion potential of steel substrates (Ecorr) shifted positively gradually and reduction of the corrosion current steadily by increasing the graphene content.

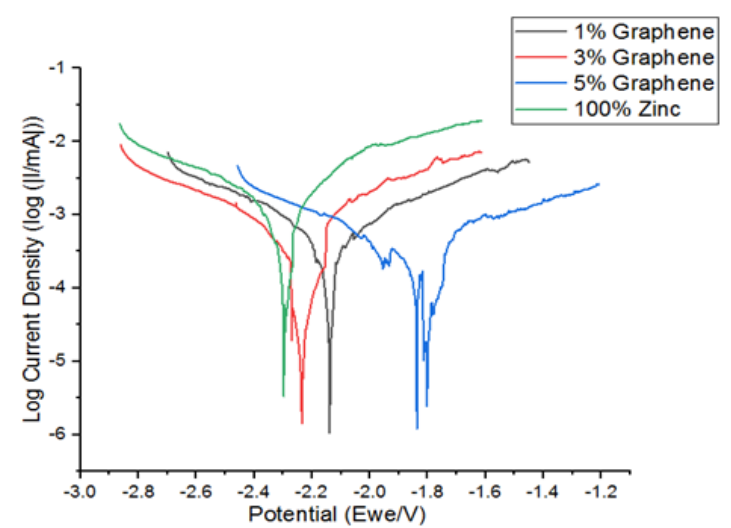

Fig. 3 Log current density log (|l/Ma|) vs potential (Ewe/V).

Additionally, the passivation capacity on the base metal surface of the passive film was stronger than the reaction of anodic passivation. Hence, the corrosion resistance for the base metal can be improve by additional of graphene, along with the graphene content of $0.5 \mathrm{wt} \%$ coating had the best resistance of corrosion. However, in the industrial environment addition of $0.1 \mathrm{wt} \%$ of Graphene with $99.9 \mathrm{wt} \%$ of Zinc in a $240 \mu \mathrm{m}$ coating layer would be sufficient as the corrosion rate is $0.0087204 \mathrm{mmpy}$ in which three times lower compared to $100 \mathrm{wt} \%$ of Zinc. Practically, $0.5 \mathrm{wt} \%$ of Graphene with $99.5 \mathrm{wt} \%$ of Zinc has the lowest corrosion rate, but the cost of $0.5 \mathrm{wt} \%$ of Graphene is high when compared to $0.1 \mathrm{wt} \%$ of Graphene.

Table 1 Test Results for coating polarization curves.

\begin{tabular}{lcccc}
\hline Reference & $\begin{array}{c}\text { 100wt\% } \\
\text { Zinc }\end{array}$ & $\mathbf{0 . 1 w t \% ~ G}$ & $\mathbf{0 . 3 w t} \%$ G & $\mathbf{0 . 5 w t \% ~ G}$ \\
\hline Ecorr (mV) & -2294.521 & -2133.348 & -2245.345 & -1799.54 \\
Icorr (uA) & 0.823 & 0.297 & 0.233 & 0.096 \\
Beta C (mV) & 356.9 & 327.2 & 270.6 & 329.0 \\
$\begin{array}{l}\text { Beta A (mV) } \\
\text { Chi }\end{array}$ & 250.4 & 333.8 & 216.5 & 146.3 \\
& 11574.3 & 1486.95 & 18124.5 & 280419.1 \\
Chi / Sqrt(N) & 4.8065 & 1.72278 & 6.0147 & 74.8144 \\
$\begin{array}{l}\text { Corrosion Rate } \\
\text { (mmpy) }\end{array}$ & 0.0241656 & 0.0087204 & 0.0068415 & 0.0028183 \\
\hline
\end{tabular}

Analysis of Electrochemical Impedance Spectroscopy (EIS)

Assumption for the uniformity of electrolyte in the coating system and even distribution on the interface of base metal of electrochemical reaction corrosion considered the reaction of the corrosion can be the caused by penetrating the electrolyte solution to interface of base metal.

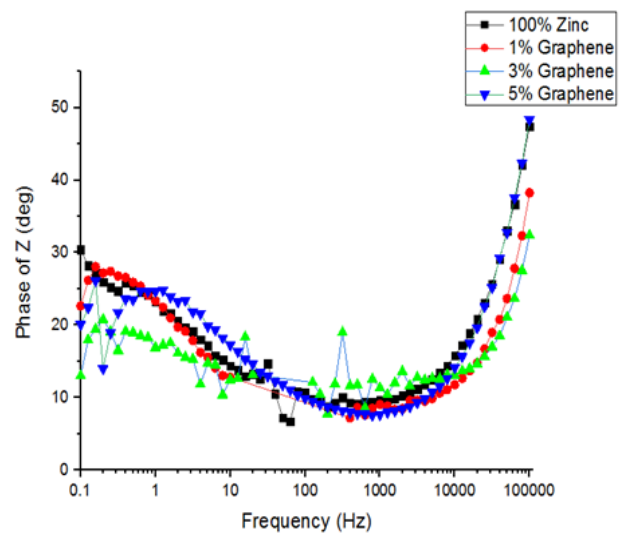

Fig. 4 Potential (V) vs elapsed time (s).

Characterization of two capacitive loops is shown in Fig. 4 where the contributions of film surface to the impedance system corresponded by the high-frequency loop while the metal contributions corresponded by the low-frequency loop. When the time constant for processes of low and high frequency nearer to each other, a larger loop of high frequency plotted for coating of $0.5 \mathrm{wt} \%$ graphene with $240 \mu \mathrm{m}$ sample of carbon steel.

The retardation for diffusion of oxygen and improvement for resistance of corrosion along with avoidance of water uptake caused by the impermeability and hydrophobic nature. On the other hand, there were significant increase in Rt value samples of the graphenecoated in addition to resistance of corrosion for substrate of carbon steel. Also, it is quite obvious from the table that higher resistance of corrosion exhibited from the sample of graphene coated.

\section{CONCLUSION}

With the rising of graphene content, the OCP number of the coating numerical also increases. This shows that reduced trend of 
thermodynamic reaction of electrochemical corrosion will be less possible to corrode. The lifting space was limited due to graphene cost and coating layer even though the coating content of $0.5 \mathrm{wt} \%$ in $240 \mu \mathrm{m}$ was slightly better. From the EIS fitting results, the addition of graphene increases impermeability of the composite coating. The integration of graphene excellently condensed the coating porosity of corrosion and improve the corrosion resistance using the characteristic frequency method. The EIS and polarization data shown have verified that by integrating different weight ratios of graphene can develop coatings for graphene-based anti-corrosive of carbon steel over a costeffective, industrial scalable method.

\section{ACKNOWLEDGEMENT}

The authors also would like to thank the Ministry of Higher Education, Malaysia for supporting this research under the Fundamental Research Grant Scheme (FRGS) Vot Number R.J130000.7851.5F077.

\section{REFERENCES}

[1] Radoman, T. S., Dzunuzovic, J. V., Jeremic, K. B., et al. 2014 Improvement of epoxy resin properties by incorporation of $\mathrm{TiO} 2$ nanoparticles surface modified with gallic acid esters. J. Mater. Des., 62:158-167.

[2] Hao, Y., Liu, F., Han, E. H. 2013, Protection of epoxy coatings containing polyaniline modified ultra-short glass fibers. J. Prog. Org. Coat., 76: 571-580.

[3] Pour-Ali, S., Dehghanian, C., Kosari, A. 2014, 'In situ synthesis of polyaniline-camphor sulfonate particles in an epoxy matrix for corrosion protection of mild steel in $\mathrm{NaCl}$ solution. J. Corros. Sci., 85: 204-214.

[4] Chmielewska, D., Sterzynski, T., Dudziec, B. 2014, Epoxy composites cured with aluminosilsesquioxanes: Thermomechanical properties. $J$. Appl. Polym. Sci., 131: 40672.

[5] Urbaniak, M. 2011, A relationship between the glass transition temperature and the conversion degree in the curing reaction of the EPY (R) epoxy system. Polymer. J, 56: 240-243.

[6] Oleksy, M., Oliwa, R., Heneczkowski, M., et al. 2012, Composites of epoxy resin with modified bentonites for aviation industry. J. Polymer, 57: 228-235.

[7] Liao, Z., Zhang, T., Qioa, S., Zhang, L. 2017, Preparation and electrochemical properties of graphene/ epoxy resin composite coating. IOP Conf. Series: Earth and Environmental Science.

[8] Ilkhani, Ahamed. 2013, Corrosion protection of carbon steel by using Zinc-Rich inorganic water-based silicate coating comprising different amounts of nano-silica. International Journal of Chemistry, 75-82.
[9] Akinci, A., Yilmaz, F. 2011, The effect of epoxy polyester sealing of sprayed-metal coatings for additional corrosion protection. AntCorrosion Methods and Materials, 26-30.

[10] El-Shazly, A. H., Al Turaif, H. A. 2012, Improving the corrosion resistance of buried steel by using polyaniline coating. International Journal of Electro-Chemical Science, 211-221.

[11] Novoselov, K. S., Geim, A. K., Morozov, S. V., et al. 2004, Electric field in atomically thin carbon films. Science, 306(5696): 666-669.

[12] Georgakilas, V., Perman, J. A., Tucek, J., Zboril, R. 2015, Broad family of carbon nanoallotropes: Classification, chemistry, and applications of fullerenes, carbon dots, nanotubes, graphene, nanodiamonds, and combined superstructures. Chem Rev., 115:4744-4822.

[13] Slonczewski, J., Weiss, P. 1958, Band structure of graphite. Phys. Rev. 109:272.

[14] Singh, V., Joung, D., Zhai, L., Das, S., Khondaker, I., Seal, S. 2011, Graphene-based materials: Past, present and future. Prog. Mater Sci., 56(8): 1178-1271.

[15] Geim, A. K., Novoselov, K. S. 2007, The rise of graphene. Nat. Mater., 6, 183-190.

[16] Pradip, G., Soga, T., Tanemura, M., Zamri, M., Jimbo, T., Katoh, R., Sumiyama, K. 2009, Vertically aligned carbon nanotubes from natural precursors by spray pyrolysis method and their field emission properties. Journal Applied Physics A., 94;1, pp. 51-56.

[17] Xingyu, W., Xiaoning, Q., Zhibin, L., Dante B. 2018, Graphene reinforced composites as protective coatings for oil and gas pipelines. Nanomaterials (Basel), 8(12):1005.

[18] Chiong, S., Goh, P., Ismail A. 2017, Novel hydrophobic PVDF/APTESGO nanocomposite for natural gas pipelines coating. J. Nat. Gas Sci. Eng., 42:190-202.

[19] Samimi, A. 2012, Use of polyurethane coating to prevent corrosion in oil and gas pipelines transfer. Int. J. Innov. Appl. Stud., 1:186-193, 2028-932.

[20] Matjaz F., Jennifer J. 2014, Application of corrosion inhibitors for steels in acidic media for the oil and gas industry: A Review. Corrosion Science, 86;17-41.

[21] Roseman, M., Martin, R., Morgan, G. 2016, Composites in offshore oil and gas applications. Marine Applications of Advanced FibreReinforced Composites, 233-257.

[22] Wang, D., Bierwagen, G. P. 2009, Sol-gel coatings on metals for corrosion protection. Ptograss in Organic Coatings, 64;4, 327-338.

[23] Gray, J. E., Luan, B. 2002, Protective coatings on magnesium and its alloys-a critical review. Journal of Alloys and Compounds, 336;1-2, 88113 . 